\title{
GABAergic ventrolateral pre-optic nucleus neurons are involved in the mediation of the anesthetic hypnosis induced by propofol
}

\author{
JIE YUAN $^{1,2}$, ZHUXIN LUO $^{2}$, YU ZHANG $^{2}$, YI ZHANG $^{1,2}$, YUAN WANG $^{1,2}$, SONG CAO $^{2,3}$, \\ BAO FU ${ }^{2,4}, \mathrm{HAO}_{\mathrm{YANG}^{2}}$, LIN ZHANG ${ }^{2}$, WENJING ZHOU ${ }^{2}$ and TIAN YU ${ }^{2}$ \\ ${ }^{1}$ Department of Anesthesiology, Affiliated Hospital of Zunyi Medical College; ${ }^{2}$ Guizhou Key Laboratory of Anesthesia \\ and Organ Protection, Zunyi Medical College; ${ }^{3}$ Department of Pain, Affiliated Hospital of Zunyi Medical College; \\ ${ }^{4}$ Department of Intense Care Unit, Affiliated Hospital of Zunyi Medical College, Zunyi, Guizhou 563000, P.R. China
}

Received February 9, 2017; Accepted July 13, 2017

DOI: $10.3892 / \mathrm{mmr} .2017 .7035$

\begin{abstract}
Intravenous anesthetics have been used clinically to induce unconsciousness for seventeen decades, however the mechanism of anesthetic-induced unconsciousness remains to be fully elucidated. It has previously been demonstrated that anesthetics exert sedative effects by acting on endogenous sleep-arousal circuits. However, few studies focus on the ventrolateral pre-optic (VLPO) to locus coeruleus (LC) sleep-arousal pathway. The present study aimed to investigate if VLPO is involved in unconsciousness induced by propofol. The present study additionally investigated if the inhibitory effect of propofol on LC neurons was mediated by activating VLPO neurons. Microinjection, target lesion and extracellular single-unit recordings were used to study the role of the VLPO-LC pathway in propofol anesthesia. The results demonstrated that $\mathrm{GABA}_{\mathrm{A}}$ agonist (THIP) or $\mathrm{GABA}_{\mathrm{A}}$ antagonist (gabazine) microinjections into VLPO altered the time of loss of righting reflex and the time of recovery of righting reflex. Furthermore, propofol suppressed the spontaneous firing activity of LC noradrenergic neurons. There was no significant difference observed in firing activity between VLPO sham lesion and VLPO lesion rats. The findings indicate that VLPO neurons are important in propofol-induced unconsciousness, however are unlikely to contribute to the inhibitory effect of propofol on LC spontaneous firing activity.
\end{abstract}

\section{Introduction}

Approximately ten million patients receive general anesthesia for surgery in China every year. While intravenous anesthetics cause unconsciousness, the mechanism and neural basis of unconsciousness are poorly understood (1). At the molecular

Correspondence to: Professor Tian Yu, Guizhou Key Laboratory of Anesthesia and Organ Protection, Zunyi Medical College, 201 Dalian Road, Zunyi, Guizhou 563000, P.R. China

E-mail: zytianyu@126.com

Key words: locus coeruleus, ventrolateral preoptic nucleus, propofol, consciousness, electrophysiology level, there are dozens of molecules known to be general anesthetic targets, including a number of ion channels (2), gap-junction channels (3), and G protein-coupled receptors (4). It's remarkable that there is no single molecular target shared by all general anesthetics (1). Therefore, effects of general anesthetics must be comprehended in the context of network connectivity.

There are similarities between general anesthesia and natural sleep. Imaging studies have shown some parallels between the anesthetized brain and the brain during deep non-rapid-eye-movement (NREM) sleep $(5,6)$. Electroencephalogram (EEG) studies have suggested that loss of consciousness caused by general anesthetics resembles the rapid transition from normal wakefulness to sleep (7). Sleep-related EEG waves that resembled gamma, delta and spindle waves have been observed during general anesthesia (8). These findings led an increasingly popular theory that anesthetics may induce unconsciousness by acting on endogenous sleep-arousal neural circuitry. But it remains unclear to what extent sleep-arousal pathway, such as the ventrolateral preoptic nucleus (VLPO)-locus coeruleus (LC) are involved in generating the hypnotic state.

Commonly used general anesthetic propofol exerts sedative effects by targeting $\mathrm{GABA}_{\mathrm{A}}$ receptors. And GABA is the primary inhibitory neurotransmitter released by sleep-promoting neurons in the VLPO, which plays a critical role in inducing and maintaining sleep (9). The VLPO sends GABAergic inhibitory projections to several wake-promoting nuclei throughout the neuroaxis (10), including the LC, tuberomammillary nucleus (TMN) and orexinergic neurons in the lateral hypothalamus (11). Previous research has demonstrated that propofol and various barbiturates activate sleep-promoting VLPO neurons through different receptors (12-14). Moreover, the inhalational anesthetic isoflurane directly depolarizes VLPO neurons (15). Nevertheless, lesion of VLPO neurons could be expected to produce resistance to anesthesia because of the accrual of sleep debt (16). Thus, it remains controversial whether VLPO activation contributes to anesthetic-induced unconsciousness.

In the present study, we hypothesized that propofol may act on VLPO neurons to stimulate the release of GABA, thereby inhibiting wakefulness-promoting neurons in the LC. 
To validate the hypothesis, we examined the LoRR (loss of righting reflex) and RoRR (recovery of righting reflex) time following $\mathrm{GABA}_{\mathrm{A}}$ receptor agonist or antagonist microinjection. We also recorded the firing activities of LC neurons under different concentrations of propofol. The results demonstrated that spontaneous firing of LC noradrenergic neurons was inhibited by propofol. Furthermore, the results also showed that firing activities were not significantly different between sham-lesion and VLPO lesion rats. Suggesting that VLPO neurons were not likely involved in propofol-mediated inhibition on LC neurons.

\section{Materials and methods}

Animals. Male Sprague-Dawley rats weighing 260-300 g $(\mathrm{n}=72)$ were housed in an isolated chamber at $20-22^{\circ} \mathrm{C}$ under a $12 \mathrm{~h}$ light/dark cycle (lights on at 8:30 AM). Food and water were available ad libitum. Twenty-four rats were used for righting reflex behavioral assays, and the remaining rats were used for in vivo electrophysiological recordings. Forty-eight neurons were recorded from forty-eight rats. All animals were supplied by the laboratory animal center of the Third Military Medical University (Chongqing, China). Animal experiments were approved by the Zunyi Medical College Animal Care and Use Committee (approval number: 2016126). All efforts were made to minimize the number of rats and their suffering.

Surgical procedures and cannulas implantation. For behavioral experiments, rats were anesthetized with sodium pentobarbital $[50 \mathrm{mg} / \mathrm{kg}$, intraperitoneally (i.p.)] and atropine sulfate $(0.2 \mathrm{mg}$, i.p.). Anesthetized animals were mounted onto a stereotaxic device (68505; RWD Life Science, China) in a flat-skull position. The core body temperature was maintained at $37-38^{\circ} \mathrm{C}$ using a heat-controlled pad equipped with a rectal probe (Stoelting Co., Wood Dale, IL, USA). Sterilized guide cannulas were implanted as described previously $(17,18)$. A pair of sterilized guide cannulas made of 23G stainless steel tubes and plugged internally with $30 \mathrm{G}$ stylets were stereotaxically implanted $2.0 \mathrm{~mm}$ above the VLPO (anteroposterior, $-0.35 \mathrm{~mm}$; left-right, $\pm 1.2 \mathrm{~mm}$; dorsoventral, $-7.0 \mathrm{~mm}$ from bregma). Stereotaxic positioning was defined according to the brain atlas of Paxinos and Watson (2007). Guide cannulas were fixed to the skull using dental cement.

Lesion formation by ibotenic acid and saline injection. To induce lesions in the VLPO, rats were anesthetized and kept in place as mentioned above. The skull of each rat was exposed, and a glass micropipette (10-12- $\mu \mathrm{m}$ tip diameter) was lowered into the VLPO region stereotaxically. Fifteen nanoliters of ibotenic acid solution (10 nmol; Sigma, St. Louis, MO, USA) $(n=24)$ or saline $(n=24)$ were injected into the VLPO bilaterally using a microinjection injector (Nanoliter 2000, World Precision Instruments, Sarasota, FL, USA) as described previously (16). The glass micropipette was slowly withdrawn after $5 \mathrm{~min}$. The two holes above VLPO were filled with bone wax. The wound was stitched with sutures and closed with wound clips. A prophylactic dose of penicillin $(50 \mathrm{ku} / \mathrm{kg}$, intramuscular injection) was injected into each rat following surgery. After seven days of recovery, in vivo electrophysiology experiments were conducted.
Righting reflex behavioral assays. In rodents, loss of consciousness can be measured by the LoRR and resumption of consciousness can be analyzed by the RoRR $(19,20)$. Fig. 1A illustrates the experimental design used to quantify the LoRR and RoRR after the VLPO was microinjected with artificial cerebrospinal fluid (ACSF) $\left(126 \mathrm{mM} \mathrm{Na}^{+}, 3 \mathrm{mM} \mathrm{K}^{+}\right.$, $\left.2 \mathrm{mM} \mathrm{Ca}^{2+}, 1.2 \mathrm{mM} \mathrm{Mg}^{2+}, 150 \mathrm{mM} \mathrm{Cl}^{-}, \mathrm{pH}=7.4\right)$ and a bolus injection of propofol $(11 \mathrm{mg} / \mathrm{kg}$, i.v.). After seven days of recovery from cannulas implantation surgery, rats $(n=24)$ were put into a Plexiglas inhalation anesthesia induction chamber for 10-min acclimation (Fig. 1A; $\mathrm{t}=-40 \mathrm{~min}$ ). Then, oxygen containing $2 \%$ isoflurane was introduced into the chamber at $2 \mathrm{l} / \mathrm{min}$ (Fig. 1A; $\mathrm{t}=-30 \mathrm{~min}$ ). The $30 \mathrm{G}$ stylet from the guide cannula was removed from the isoflurane-anesthetized rats, and a $31 \mathrm{G}$ injection cannula was inserted. An injection cannula was attached to a dual-channel microinjection syringe with PE tubing under the control of an injection pump (302; RWD Life Science), and then a $24 \mathrm{G}$ intravenous catheter was implanted and fixed to the rat's tail vein. Isoflurane administration was subsequently discontinued. The ACSF ( $\mathrm{t}=0 \mathrm{~min} ; 0.2 \mu \mathrm{l} / \mathrm{side}$; $\mathrm{n}=24$ ) (Fig. 1A) was microinjected into the bilateral VLPO at an infusion speed of $0.2 \mu \mathrm{l} / \mathrm{min}$ for $1 \mathrm{~min}(\mathrm{t}=1$ to $\mathrm{t}=2$ ) (Fig. 1A). Five min later ( $\mathrm{t}=7 \mathrm{~min})$ (Fig. 1A), propofol $(11 \mathrm{mg} / \mathrm{kg}, \mathrm{n}=24)$ was bolus injected into the tail vein. The induction time of propofol was quantified as the time (sec) to LoRR; the resumption time was quantified as the time to RoRR (sec). Three days later, the twenty-four rats were subdivided into $\mathrm{GABA}_{\mathrm{A}}$ agonist group $(n=12)$ and $\mathrm{GABA}_{\mathrm{A}}$ antagonist group $(\mathrm{n}=12)$. Rats in the agonist group were microinjected with the $\mathrm{GABA}_{\mathrm{A}}$ agonist 4,5,6,7-tetrahydroisoxazolo (5,4-c) pyridin-3-ol (THIP), also called Gaboxadol $(t=0 ; n=12)$ (Fig. 1B). Rats in the $\mathrm{GABA}_{\mathrm{A}}$ antagonist group received the same dose of gabazine $(t=0$; $\mathrm{n}=12$ ) (Fig. 1C), and the timeline and procedures were the same as in the ACSF group.

Extracellular single-unit recordings. After a recovery period of seven days, the extracellular single-unit recording was performed from noradrenergic neurons of the LC (LC-NA) with VLPO lesions $(n=24)$ and sham lesions $(n=24)$ rats. Each group was subdivided into a consciousness group ( $\mathrm{n}=6$, without propofol administration), a low propofol concentration group ( $\mathrm{n}=6 ; 20 \mathrm{mg} / \mathrm{kg} / \mathrm{h}$, i.v.), medium concentration group $(\mathrm{n}=6 ; 40 \mathrm{mg} / \mathrm{kg} / \mathrm{h}$, i.v.) and a high concentration propofol group ( $\mathrm{n}=6 ; 60 \mathrm{mg} / \mathrm{kg} / \mathrm{h}$, i.v.).

The protocol of extracellular single-unit recordings in non-anesthetized rats was refereed to previously report (21). Briefly, the rats were progressively habituated to the head-restrained position (7-14 days) by placing them inside a plastic chamber, painlessly restraining the head with a head holder and preventing large body movements with a cotton-coated plastic cover. The eyes were covered to reduce visual stimulation. After 7-14 days of habituation, the rats could be kept in a sphinx position for 3-6 consecutive $h$ without showing any signs of discomfort. If any signs of discomfort were seen, the rats were freed from the restrained position.

Then A 24G intravenous catheter was implanted and fixed as described above. Rats were removed from the induction chamber and mounted onto a stereotaxic device with blunt ear bars. Propofol was continuously delivered via a syringe pump (WZS-50F6; Smith Medical, Plymouth, MN, US) connected 
A

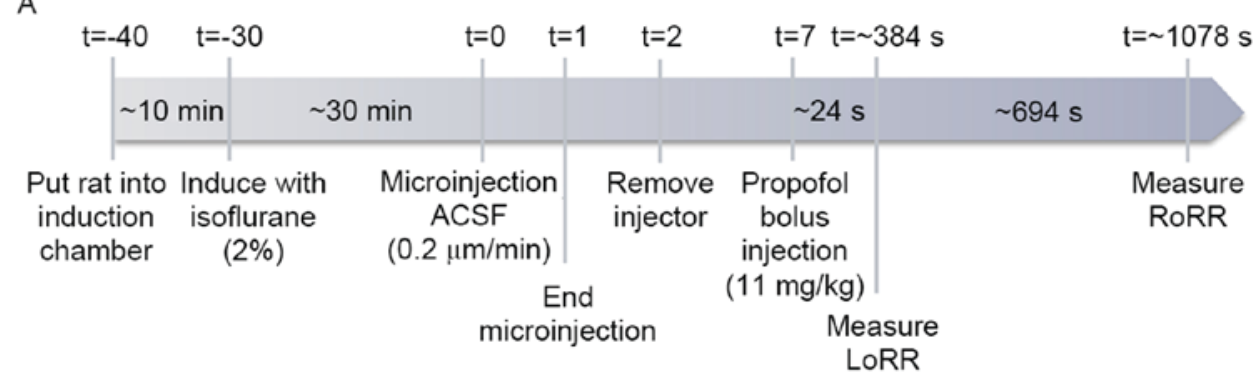

B

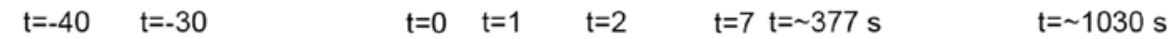

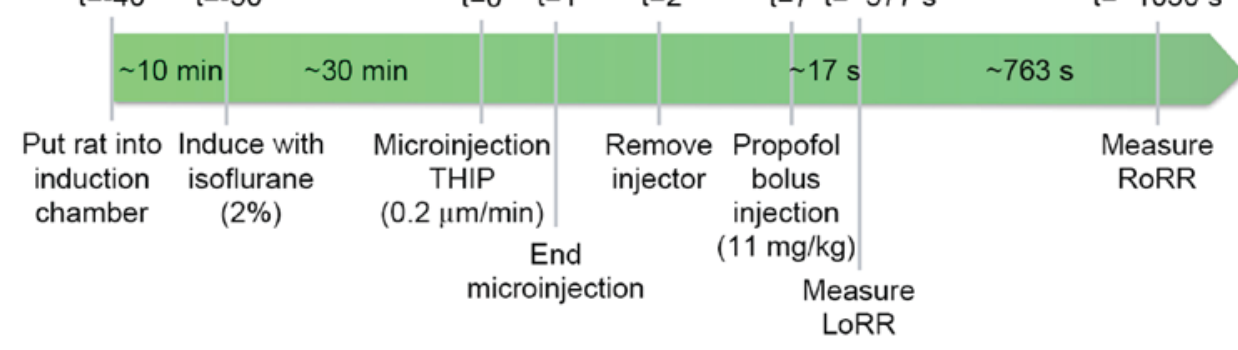

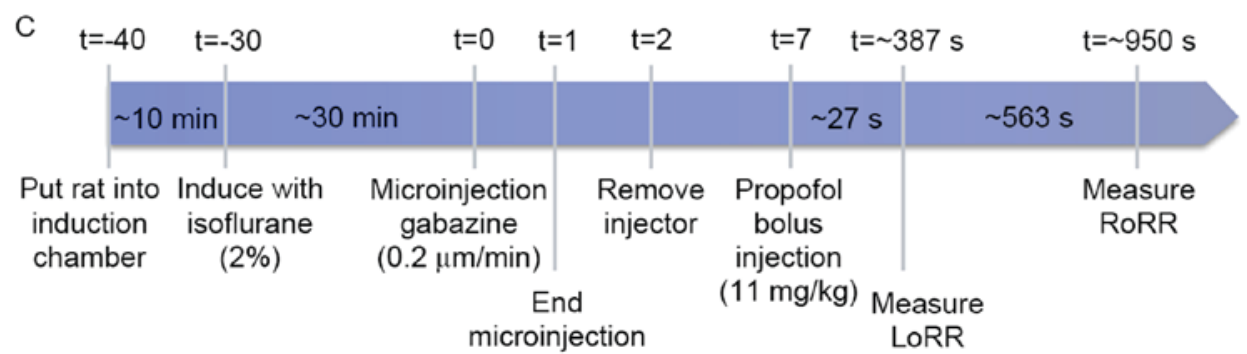

Figure 1. (A) Timelines of LoRR and RoRR measurement. Rats were left for $30 \mathrm{~min}$ after isoflurane administration for the righting reflex to recover before microinjection of ACSF (vehicle control), THIP (B), and gabazine (C) into the VLPO and before propofol bolus injection. ACSF (A), THIP (B), and gabazine (C) were microinjected while the rats were awake. Five min after injection, propofol was bolus injected (11 mg/kg) into the tail vein. The LoRR recorded and quantified immediately. Then, rats were placed in a supine position and the RoRR was measured. All experimental procedures were the same in the agonist (B), antagonist (C), and control (A) groups except for the microinjection of drugs. LoRR, loss of righting reflex; RoRR, resumption of righting reflex; ACSF, artificial cerebrospinal fluid.

to the intravenous catheter in propofol administration groups. LC-NA extracellular recordings were obtained as previously described (21). A single barrel glass micropipette was filled with $0.5 \%$ sodium acetate and lowered into the LC $(3.5 \mathrm{~mm}$ caudal to lambda, 0.85-1.0 mm lateral to the midline, 5-6.5- $\mathrm{mm}$ below the cortical surface) by using a micromanipulator (Mini 25; Luigs \& Neumann, Ratingen, Germany) according to coordinates defined in the brain atlas of Paxinos and Watson (2007). The impedance of the micropipette was 10-20 M $\Omega$, measured in physiological saline. Recording was performed during a period of at least $5 \mathrm{~min}$, with only one cell measured from each rat. The recorded extracellular signals were amplified using a patch clamp amplifier (EPC10; HEKA Elektronik, Lambrecht, Germany), bandpass filtered (100-10,000 Hz) and saved to a computer installed with PATCHMASTER (Heka) and Mini Analysis System (Synaptosoft, Inc., Fort Lee, NJ, USA) for offline analysis. The body temperature of rats was maintained at $37-38^{\circ} \mathrm{C}$ during the recording period.

Noxious cutaneous stimulation. The discharge patterns of NA-LC neurons were identified using standard criteria $(22,23)$. They display a positive-negative action potential shape with a notch on the ascending limb (Fig. 2A) and a typical, biphasic excitation-inhibition response to noxious stimulation of the contralateral hind paw (Fig. 2B). We pinched the skin of the rat's hind paw using toothed forceps for cutaneous nociceptive stimulation to induce this characteristic firing response. The noxious stimulation procedure was only operated in anesthetized rats.

Histological localization of microinjection and lesion areas. After behavioral and electrophysiology experiments, rats were deeply anesthetized with sodium pentobarbital. Cold $100 \mathrm{ml}$ physiological saline was perfused through the ascending aorta, followed by $200 \mathrm{ml} 4 \%$ paraformaldehyde. The brains were then removed, post-fixed for $24 \mathrm{~h}$ at $4^{\circ} \mathrm{C}$ in paraformaldehyde, and transferred to glass containers filled with $30 \%$ sucrose for $48 \mathrm{~h}$ at $4^{\circ} \mathrm{C}$. $20-\mu \mathrm{m}$ coronal sections containing microinjection or lesion sites were cut and stained with hematoxylin-eosin for histological verification (Fig. 3). Stereotaxic coordinates were identified according to the brain atlas of Paxinos and Watson (2007).

Statistical analyses. Statistical analyses were performed using SPSS 17.0 for Windows. All data were expressed as means \pm standard deviation. A P-value of $<0.05$ was considered statistically significant using paired-samples t-test in behavioral experiments. $\mathrm{N}$ refers to the number of rats studied. The 
A

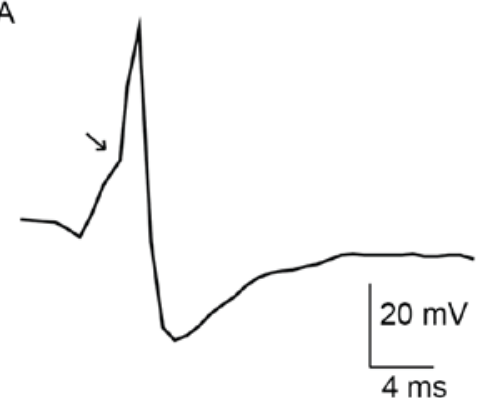

B

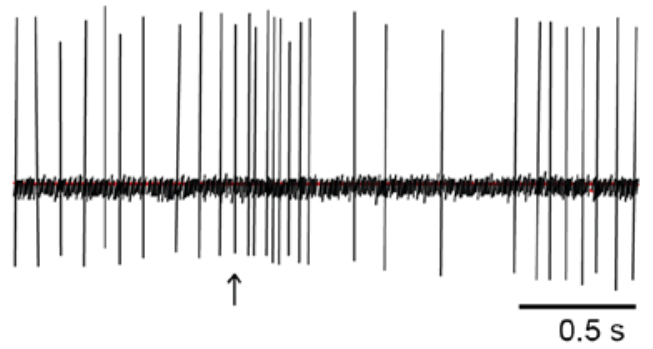

Figure 2. In vivo extracellular recording of action potentials in noradrenergic LC neurons. (A) the positive-negative waveform firing of a typical LC noradrenergic neuron. The arrow indicates the notch on the ascending limb. (B) the characteristic firing pattern of an LC neuron in response to pinch stimulation of the contralateral hind paw, showing a biphasic response with excitation followed by inhibition. The arrow indicates when the pinch was applied. Bar, $0.5 \mathrm{~s}$.
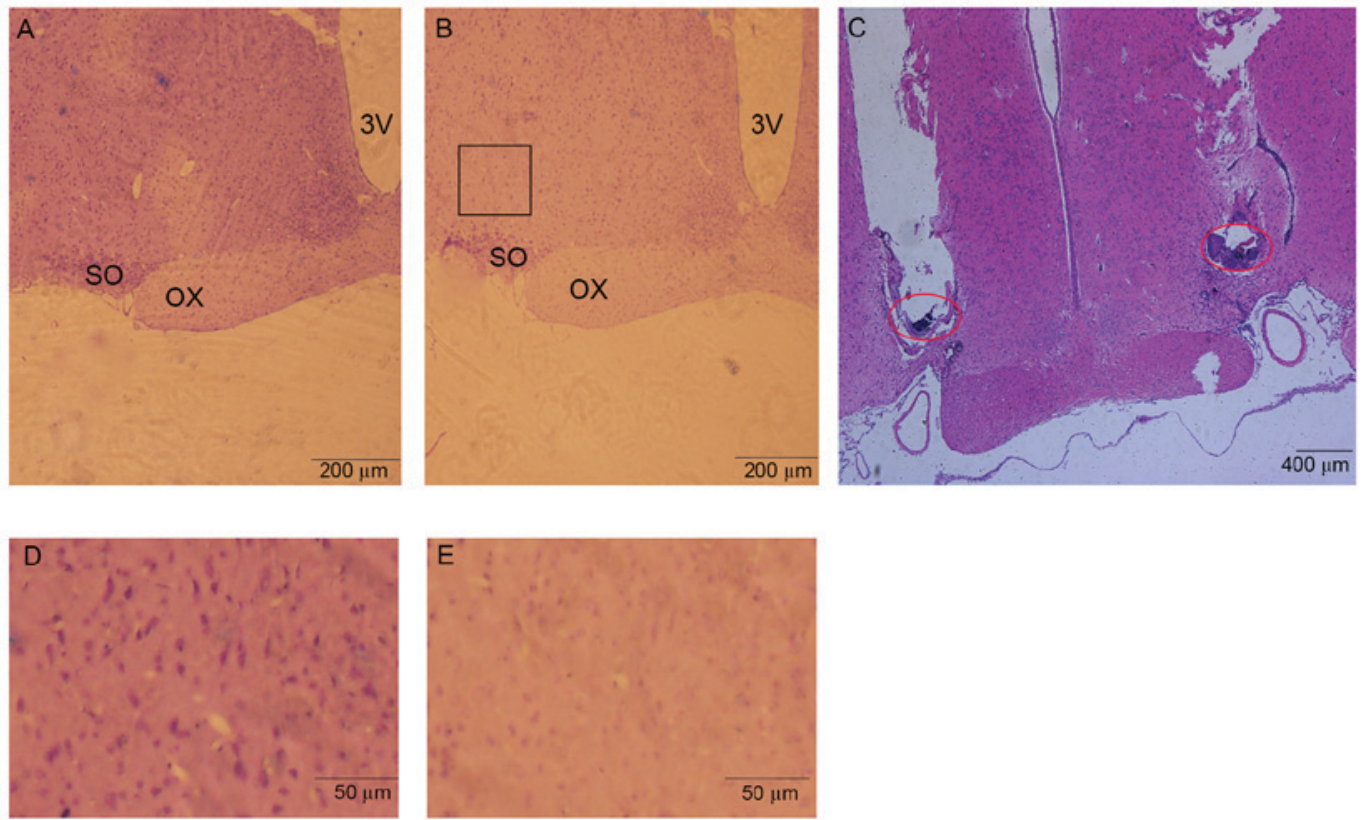

Figure 3. Photomicrographs illustrating the sham-lesion and lesion in the VLPO, and the localization of the cannula tip. (A) shows the sham-lesion induced by saline, (B) shows a lesion induced by ibotenic acid in the VLPO (outline by a black square), magnification x40. (C) Shows the localization of the microinjection cannula tip in the bilateral VLPO (hematoxylin \& eosin x10). The red circle shows the tip of microinjection cannula. (D and E) Shows the magnification pictures in sham-lesion VLPO and lesion VLPO areas x100. Sections were stained with hematoxylin-eosin. 3V, third ventricle; OX, optic chiasm; SO, supraoptic nucleus.

firing frequencies of noradrenergic neurons in different groups were compared using independent-samples t-test. The firing frequencies of noradrenergic neurons in the same group were compared using one-way ANOVA, and n refers to the number of neurons analyzed.

\section{Results}

The effects of $G A B A_{A}$ receptors in the VLPO on LoRR and $R o R R$. To determine whether GABAergic neurons are involved in unconsciousness induced by propofol. We microinjected $\mathrm{GABA}_{\mathrm{A}}$ agonist (THIP) or GABA $\mathrm{A}_{\mathrm{A}}$ antagonist (gabazine) into VLPO and observed the LoRR and RoRR. Fig. 4A and B illustrates how ACSF and THIP microinjection into the VLPO of the same rats $(n=12)$ affected propofol-mediated anesthesia and subsequent recovery. Microinjection of THIP into the VLPO decreased LoRR $(23.67 \pm 8.24 \mathrm{sec}$ to $16.83 \pm 6.41 \mathrm{sec}$, $\mathrm{P}<0.05)$ (Fig. 4A) and increased RoRR $(694.00 \pm 126.73 \mathrm{sec}$ to
$762.83 \pm 126.28 \mathrm{sec}, \mathrm{P}<0.05$ ) (Fig. 4B) before propofol administration. (Fig. 4C and D) illustrates the effects of ACSF and the $\mathrm{GABA}_{\mathrm{A}}$ antagonist gabazine on propofol-mediated anesthesia and recovery in the same rats $(n=12)$. Microinjection of gabazine into the VLPO increased LoRR $(20.42 \pm 4.14 \mathrm{sec}$ to $26.83 \pm 6.28 \mathrm{sec}, \mathrm{P}<0.05$ ) (Fig. 4C) and decreased RoRR $(605.67 \pm 81.79 \mathrm{sec}$ to $562.58 \pm 78.05 \mathrm{sec}, \mathrm{P}<0.05$, Fig. 4D) before propofol administration.

The effect of propofol on the spontaneous firing activity of $L C$ neurons in rats with VLPO lesion. To explore the effect of propofol on LC firing activity, we anesthetized the VLPO sham-lesion and VLPO lesion rats with different concentrations of propofol. Fig. 5 shows that propofol inhibited the spontaneous firing activity of LC neurons in a dose-dependent manner. In the VLPO sham-lesion group, the spontaneous firing activity of LC neurons in medium propofol concentration $(40 \mathrm{mg} / \mathrm{kg} / \mathrm{h})$ was reduced to $2.98 \pm 0.6 \mathrm{~Hz}$ compared with 
A

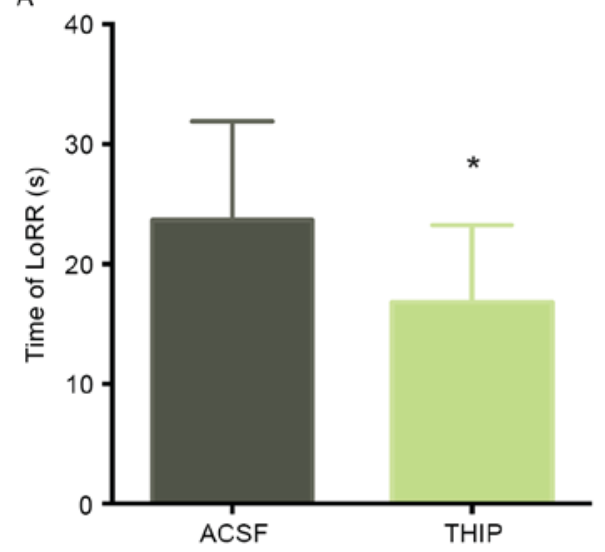

C

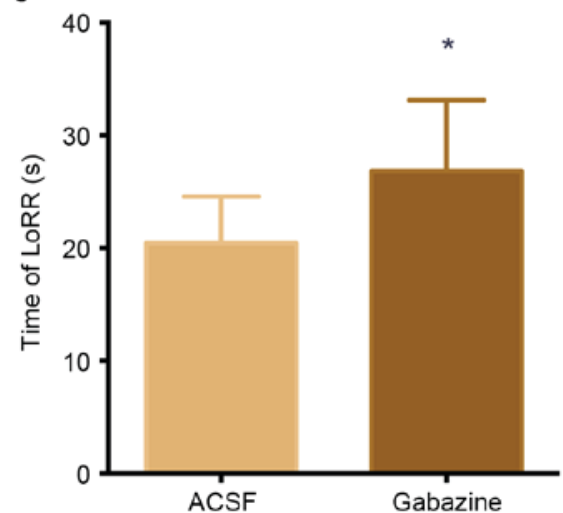

$B$

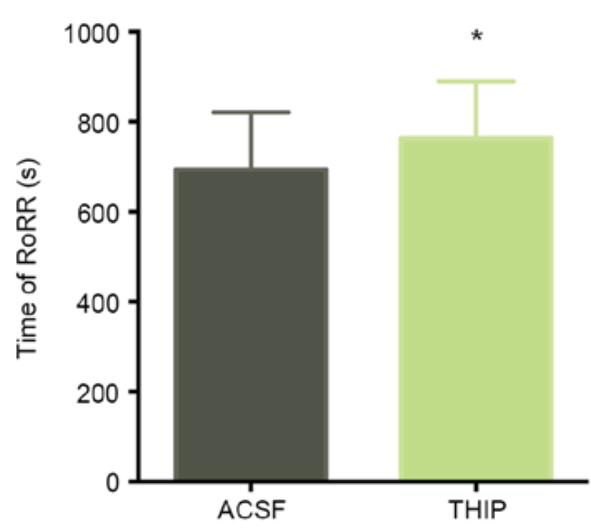

D

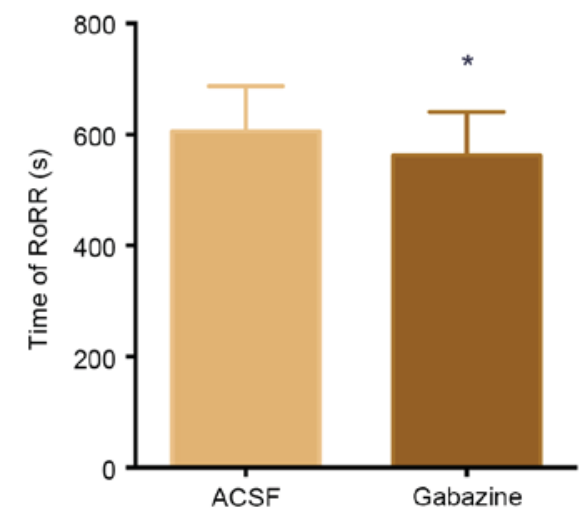

Figure 4. The effects of ACSF, THIP, and gabazine on propofol-induced anesthesia and recovery. Microinjection the GABA agonist THIP into the VLPO significantly decreased the LoRR (A) and increased the RoRR (B) after propofol administration (" $\mathrm{P}<0.05$ vs. ACSF microinjection, $\mathrm{n}=12$ ). In contrast, microinjection of the GABA $A_{A}$ antagonist significantly increased the LoRR (C) and decreased the RoRR (D) after propofol administration ("P $<0.05$ vs. ACSF microinjection, $\mathrm{n}=12$ ).

$6.54 \pm 1.16 \mathrm{~Hz}$ of the low propofol concentration $(20 \mathrm{mg} / \mathrm{kg} / \mathrm{h})$ group $(\mathrm{P}<0.05)$. The spontaneous firing activity of LC neurons in high propofol concentration $(60 \mathrm{mg} / \mathrm{kg} / \mathrm{h})$ was reduced to $1.36 \pm 0.9 \mathrm{~Hz}$ compared with $2.98 \pm 0.6 \mathrm{~Hz}$ of the medium propofol concentration $(40 \mathrm{mg} / \mathrm{kg} / \mathrm{h})$ group $(\mathrm{P}<0.05)$. The firing activity of LC neurons in medium propofol concentration was reduced to $2.98 \pm 0.6 \mathrm{~Hz}$ compared with $7.32 \pm 1.33 \mathrm{~Hz}$ of the consciousness group $(\mathrm{P}<0.05)$. And the firing activity of LC neurons in high propofol concentration was reduced to $1.36 \pm 0.9 \mathrm{~Hz}$ compared with $7.32 \pm 1.33 \mathrm{~Hz}$ of the consciousness group $(\mathrm{P}<0.05)$. However, there's no statistical significant differences in the firing activity between consciousness and low propofol concentration group, (Fig. 5A).

The similar inhibitory effect was observed in the VLPO lesion group. In this group, the spontaneous firing activity of LC neurons in medium propofol concentration rats was $2.80 \pm 0.46 \mathrm{~Hz}$ compared with $5.89 \pm 0.93 \mathrm{~Hz}$ in the low propofol concentration group $(\mathrm{P}<0.05)$. The spontaneous firing activity of LC neurons in high propofol concentration rats was $1.57 \pm 0.85 \mathrm{~Hz}$ compared with $2.80 \pm 0.46 \mathrm{~Hz}$ in the medium propofol concentration group $(\mathrm{P}<0.05)$. The firing activity of LC neurons in medium propofol concentration was reduced to $2.80 \pm 0.46 \mathrm{~Hz}$ compared with $6.99 \pm 2.03 \mathrm{~Hz}$ of the consciousness group $(\mathrm{P}<0.05)$. And the firing activity of LC neurons in high propofol concentration was reduced to $1.57 \pm 0.85 \mathrm{~Hz}$ compared with $6.99 \pm 2.03 \mathrm{~Hz}$ of the consciousness group $(\mathrm{P}<0.05)$. In addition, there's no statistical significant differences in the firing activity between consciousness and low propofol concentration group, (Fig. 5B). Nevertheless, our experimental results showed that firing activities were not significantly different between sham-lesion and VLPO lesion rats after administration of the same concentration of propofol ( $\mathrm{P}>0.05)$, (Fig. 5C).

\section{Discussion}

When we microinjected the GABA $\mathrm{A}_{\mathrm{A}}$ agonist THIP into the VLPO before propofol administration, results showed that LoRR decreased while RoRR increased. In contrast, microinjection of the $\mathrm{GABA}_{\mathrm{A}}$ antagonist gabazine into the VLPO increased LoRR and decreased RoRR. The results indicate that $\mathrm{GABA}_{\mathrm{A}}$ receptors in the VLPO are involved in propofol-induced unconsciousness, which is consistent with previous reports demonstrating that potentiating GABAergic transmission in the VLPO promotes sleep $(15,24,25)$. The VLPO was the first nucleus to be identified as a sleep-regulating center containing GABAergic neurons (26). Two-thirds of VLPO neurons are inhibited by the wake-promoting neurotransmitter noradrenaline (NA). These 


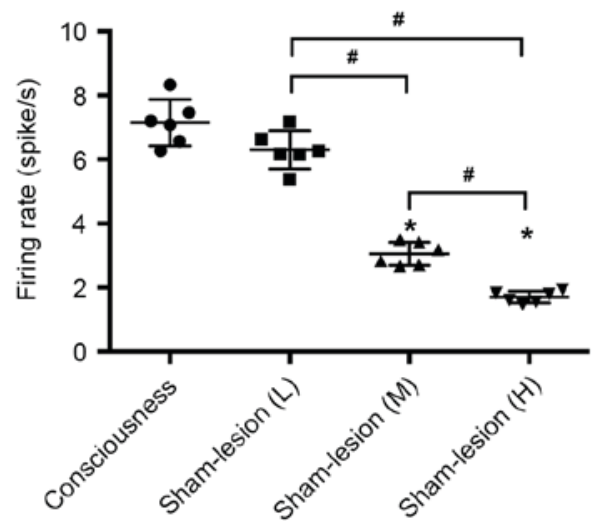

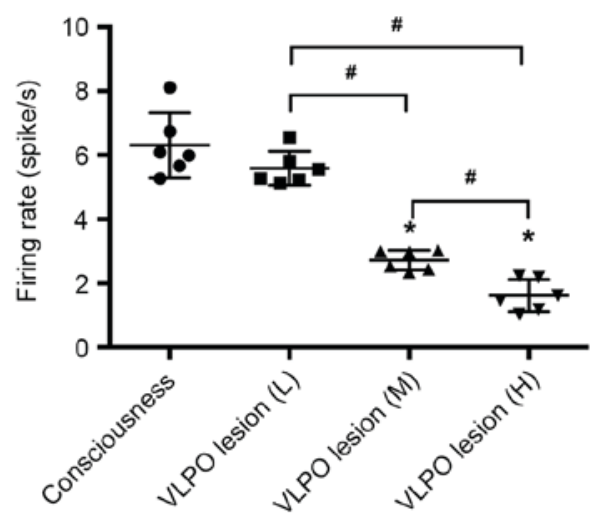

C

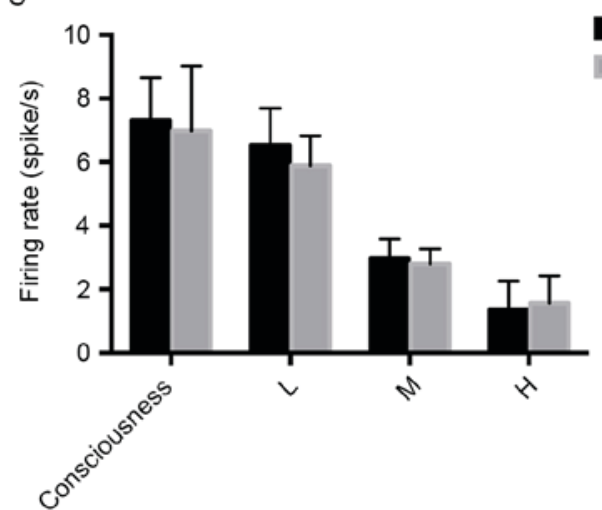

Sham-lesion

VLPO lesion

Figure 5. Effect of different propofol concentration on firing rate of LC noradrenergic neurons in VLPO sham-lesion and lesion rats. (A) Shows the low (20 mg/ $\mathrm{kg} / \mathrm{h}$, i.v.), medium ( $40 \mathrm{mg} / \mathrm{kg} / \mathrm{h}$, i.v.), high $(60 \mathrm{mg} / \mathrm{kg} / \mathrm{h}$, i.v.) propofol concentration administration and without propofol administration on LC noradrenergic neurons firing activity in VLPO sham-lesion rats. (B) Shows the low $(20 \mathrm{mg} / \mathrm{kg} / \mathrm{h}$, i.v.), medium $(40 \mathrm{mg} / \mathrm{kg} / \mathrm{h}$, i.v.), high (60 mg/kg/h, i.v.) propofol concentration administration and without propofol administration on LC noradrenergic neurons firing activity in VLPO lesion rats. (C) Shows different propofol concentration administration and without propofol administration on LC noradrenergic neurons firing activity in VLPO sham-lesion and lesion rats. ${ }^{~} \mathrm{P}<0.05 \mathrm{high}$ propofol concentration group vs. medium propofol concentration group, $\mathrm{n}=6$; medium propofol concentration group vs. low propofol concentration group, $\mathrm{n}=6$. ${ }^{*} \mathrm{P}<0.05$ medium propofol concentration vs. consciousness group, $\mathrm{n}=6$; high propofol concentration vs. consciousness group, $\mathrm{n}=6$. $\mathrm{L}$, low propofol concentration administration (20 mg/kg/h, i.v.); $\mathrm{M}$, medium propofol concentration administration ( $40 \mathrm{mg} / \mathrm{kg} / \mathrm{h}$, i.v.); H, high propofol concentration administration (60 mg/kg/h, i.v.).

are putative sleep-promoting neurons and can be activated by general anesthetics. The remaining one-third of neurons can be excited by NA and inhibited by general anesthetics (14). Patch clamp experiments (27) have shown that propofol directly activates $\mathrm{NA}(-)$ neurons via $\mathrm{GABA}_{\mathrm{A}}$ receptors. THIP is a specific $\mathrm{GABA}_{\mathrm{A}}$ receptor agonist (28) and microinjection of THIP into the pontine reticular formation promotes wakefulness (29). On the other hand, microinjection of THIP into the VLPO improves sleep (30).

Our work suggests that early activation of VLPO GABAergic neurons enhances the anesthetic effect of propofol. This effect may be the result of more GABA releasing into the arousal system in response to THIP. Nelson (12) demonstrated that the sedative effects of GABA-responsive agents were attenuated when gabazine was microinjected into the TMN nucleus. In our study, the gabazine microinjection significantly increased LoRR and decreased RoRR. After the GABA receptor was blocked, propofol-induced anesthesia was attenuated. In conclusion, potentiating GABAergic transmission in the VLPO may represent a mechanism for propofol-induced anesthesia. In contrast, blocking GABAergic transmission may attenuate unconsciousness following propofol administration.
In the present study, we also found that the firing rate of LC neurons was dramatically decreased when the concentration of propofol was higher. It was reported that the persistent inward calcium current and the cAMP-activated inward sodium current formed the spontaneous firing activity of LC neurons (31). Previously in vitro patch clamp experiments demonstrated that propofol inhibited the LC neurons by activation of outward chloride current, and the $\mathrm{GABA}_{\mathrm{A}}$ (rather than $\mathrm{GABA}_{\mathrm{B}}$ ) receptor antagonists could block the inhibitory effect (32). Accordingly, in our in vivo extracellular single-unit recordings experiments, we hypothesize that outward chloride current activated by propofol can balance the inward calcium current and the cAMP-activated sodium current. Thus, the spontaneous firing activity of LC will be inhibited. Furthermore, higher propofol concentration could increase the outward chloride current as to hyperpolarize the LC neurons.

The LC neurons fire continually during consciousness and the firing rate decreases during non-rapid eye movement (NREM) and rapid eye movement (REM) sleep (33). Halothane is an agent that could suppress such activity of LC neurons (34). Moreover, $\alpha 2$-adrenergeic receptors in the LC are a major target for the sedative agent dexmedetomidine (35), 
which directly inhibits LC neurons. Our data showed that the firing rate of LC neurons was not influenced by VLPO lesions, indicating that VLPO GABAergic neurons were not involved in the propofol-mediated inhibition on LC neurons.

VLPO lesions could induce acute sleep loss in rats $(16,36)$ and confer a short-term resistance to isoflurane (15). Our previous work (37) conducted acute lesions in VLPO culminated in opposite results, which indicated that VLPO is necessary for the propofol-induced inhibition of LC activity. Another thing to note is that sleep debt caused by sleep loss can amplify the sensitivity of propofol and isoflurane (38). In order to minimize the effect of sleep debt, we performed experiments seven days after the lesions. VLPO lesions did not affect the firing rate of LC neurons after propofol was administered. This could also be explained by the presence of different sleep-promoting neuron populations in the median preoptic nucleus (MNPO) (39), basal forebrain (BF) (40) and cortex (41). If one region is experimentally lesioned, other sleep-promoting systems remain intact. Thus, LC neurons also received inhibitory afferent projections from these nuclei, which may have compensated for the loss of VLPO GABAergic neurons in this study.

However, this study is limited in the following aspects. We aim to investigate the role NA(-) neurons play in propofol-induced anesthesia. Nevertheless, NA(+) neurons in the VLPO were also lesioned by ibotenic acid. Specific inactivation of $\mathrm{NA}(-)$ neurons by pharmacogenetics or genetic manipulation would be a better approach to conducting our study. Additionally, quantifying the changes of neurotransmitters GABA in the LC area would make our conclusion more persuasive.

In conclusion, we identified that VLPO GABAergic neurons play a crucial role in propofol-induced unconsciousness. Propofol could suppress the spontaneous firing activity of LC noradrenergic neurons in vivo, but VLPO neurons are not involved in propofol-mediated inhibition on LC neurons. Present findings are consistent with the hypothesis that general anesthetics act on endogenous sleep-wake circuitry to exert sedative effects.

\section{Acknowledgements}

This research was supported by grants from the National Natural Science Foundation of China (NO. 81460219). All work was conducted in the Guizhou Key Laboratory of Anesthesia and Organ Protection, Zunyi, China. JY, YZ and $\mathrm{BF}$ contributed to the conception and design of the work. JY, $\mathrm{ZL}$, and $\mathrm{YZ}$ performed the behavioral assays. SC, BF, and JY performed histological staining and analysis. JY, LZ, ZW and YZ were responsible for the in vivo recording. JY analyzed the data and drafted the manuscript. All authors approved the final version of the manuscript.

\section{References}

1. Brown EN, Lydic R and Schiff ND: General anesthesia, sleep, and coma. N Engl J Med 363: 2638-2650, 2010.

2. Franks NP: Molecular targets underlying general anaesthesia. $\mathrm{Br}$ J Pharmacol 147 (Suppl 1): S72-S81, 2006.

3. Yasui Y, Masaki E and Kato F: Sevoflurane directly excites locus coeruleus neurons of rats. Anesthesiology 107: 992-1002, 2007.
4. Ishizawa Y, Pidikiti R, Liebman PA and Eckenhoff RG: $\mathrm{G}$ protein-coupled receptors as direct targets of inhaled anesthetics. Mol Pharmacol 61: 945-952, 2002.

5. Braun AR, Balkin TJ, Wesenten NJ, Carson RE, Varga M, Baldwin P, Selbie S, Belenky G and Herscovitch P: Regional cerebral blood flow throughout the sleep-wake cycle. An H2(15) O PET study. Brain 120: 1173-1197, 1997.

6. Kajimura N, Uchiyama M, Takayama Y, Uchida S, Uema T, Kato M, Sekimoto M, Watanabe T, Nakajima T, Horikoshi S, et al: Activity of midbrain reticular formation and neocortex during the progression of human non-rapid eye movement sleep. J Neurosci 19: 10065-10073, 1999.

7. Boly M, Moran R, Murphy M, Boveroux P, Bruno MA, Noirhomme Q, Ledoux D, Bonhomme V, Brichant JF, Tononi G, et al: Connectivity changes underlying spectral EEG changes during propofol-induced loss of consciousness. J Neurosci 32: 7082-7090, 2012.

8. Voss L and Sleigh J: Monitoring consciousness: The current status of EEG-based depth of anaesthesia monitors. Best Pract Res Clin Anaesthesiol 21: 313-325, 2007.

9. Franks NP: General anaesthesia: From molecular targets to neuronal pathways of sleep and arousal. Nat Rev Neurosci 9: 370-386, 2008.

10. Saper CB, Scammell TE and Lu J: Hypothalamic regulation of sleep and circadian rhythms. Nature 437: 1257-1263, 2015.

11. Saper CB, Fuller PM, Pedersen NP, Lu J and Scammell TE: Sleep state switching. Neuron 68: 1023-1042, 2010.

12. Nelson LE, Guo TZ, Lu J, Saper CB, Franks NP and Maze M: The sedative component of anesthesia is mediated by GABA(A) receptors in an endogenous sleep pathway. Nat Neurosci 5: 979-984, 2002

13. Lu J, Nelson LE, Franks N, Maze M, Chamberlin NL and Saper CB: Role of endogenous sleep-wake and analgesic systems in anesthesia. J Comp Neurol 508: 648-662, 2008.

14. Li KY, Guan YZ, Krnjević K and Ye JH: Propofol facilitates glutamatergic transmission to neurons of the ventrolateral preoptic nucleus. Anesthesiology 111: 1271-1278, 2009.

15. Moore JT, Chen J, Han B, Meng QC, Veasey SC, Beck SG and Kelz MB: Direct activation of sleep-promoting VLPO neurons by volatile anesthetics contributes to anesthetic hypnosis. Curr Biol 22: 2008-2016, 2012.

16. Lu J, Greco MA, Shiromani P and Saper CB: Effect of lesions of the ventrolateral preoptic nucleus on NREM and REM sleep. J Neurosci 20: 3830-3842, 2000.

17. Zhang J, Yin D, Wu F, Zhang G, Jiang C, Li Z, Wang L and Wang K: Microinjection of adenosine into the hypothalamic ventrolateral preoptic area enhances wakefulness via the A1 receptor in rats. Neurochem Res 38: 1616-1623, 2013.

18. Sukhotinsky I, Zalkind V, Lu J, Hopkins DA, Saper CB and Devor M: Neural pathways associated with loss of consciousness caused by intracerebral microinjection of GABA A-active anesthetics. Eur J Neurosci 25: 1417-1436, 2007.

19. Tung A, Herrera S, Szafran MJ, Kasza K and Mendelson WB: Effect of sleep deprivation on righting reflex in the rat is partially reversed by administration of adenosine $\mathrm{A} 1$ and $\mathrm{A} 2$ receptor antagonists. Anesthesiology 102: 1158-1164, 2005.

20. Vanini G, Watson CJ, Lydic R and Baghdoyan HA: Gamma-aminobutyric acid-mediated neurotransmission in the pontine reticular formation modulates hypnosis, immobility, and breathing during isoflurane anesthesia. Anesthesiology 109: 978-988, 2008.

21. Takahashi K, Kayama Y, Lin JS and Sakai K: Locus coeruleus neuronal activity during the sleep-waking cycle in mice. Neuroscience 169: 1115-1126, 2010.

22. Sugiyama D, Hur SW, Pickering AE, Kase D, Kim SJ, Kawamata M, Imoto $\mathrm{K}$ and Furue $\mathrm{H}$ : In vivo patch-clamp recording from locus coeruleus neurones in the rat brainstem. J Physiol 590: 2225-2231, 2012.

23. Wang T, Zhang QJ, Liu J, Wu ZH and Wang S: Firing activity of locus coeruleus noradrenergic neurons increases in a rodent model of Parkinsonism. Neurosci Bull 25: 15-20, 2009.

24. Lydic R and Baghdoyan HA: Sleep, anesthesiology, and the neurobiology of arousal state control. Anesthesiology 103: 1268-1295, 2005

25. Xiong M, Li J, Wang D, Delphin E and Ye JH: Intra-ventrolateral preoptic nucleus injection of $\gamma$-aminobutyric acid induces sedation in rats. Int J Physiol Pathophysiol Pharmacol 4: 94-98, 2012.

26. Sherin JE, Shiromani PJ, McCarley RW and Saper CB: Activation of ventrolateral preoptic neurons during sleep. Science 271: 216-219, 1996. 
27. Liu YW, Zuo W and Ye JH: Propofol stimulates noradrenalin-inhibited neurons in the ventrolateral preoptic nucleus by reducing GABAergic inhibition. Anesth Analg 117: 358-363, 2013.

28. Boehm SL II, Homanics GE, Blednov YA and Harris RA delta-Subunit containing GABAA receptor knockout mice are less sensitive to the actions of 4,5,6,7-tetrahydroisoxazolo-[5,4-c] pyridin-3-ol. Eur J Pharmacol 541: 158-162, 2006.

29. Vanini G and Baghdoyan HA: Extrasynaptic GABAA receptors in rat pontine reticular formation increase wakefulness. Sleep 36: 337-343, 2013.

30. Lu J and Greco MA: Sleep circuitry and the hypnotic mechanism of GABAA drugs. J Clin Sleep Med 2 (Suppl): S19-S26, 2006.

31. Williams JT and North RA: Opiate-receptor interactions on single locus coeruleus neurones. Mol Pharmacol 26: 489-497, 1984.

32. Chen CL, Yang YR and Chiu TH: Activation of rat locus coeruleus neuron GABA(A) receptors by propofol and its potentiation by pentobarbital or alphaxalone. Eur J Pharmacol 386: 201-210, 1999.

33. Berridge CW and Waterhouse BD: The locus coeruleus-noradrenergic system: Modulation of behavioral state and state-dependent cognitive processes. Brain Res Brain Res Rev 42: 33-84, 2003.

34. Sirois JE, Lei Q, Talley EM, Lynch C III and Bayliss DA: The TASK-1 two-pore domain $\mathrm{K}+$ channel is a molecular substrate for neuronal effects of inhalation anesthetics. J Neurosci 20: 6347-6354, 2000.

35. Correa-Sales C, Rabin BC and Maze M: A hypnotic response to dexmedetomidine, an alpha 2 agonist, is mediated in the locus coeruleus in rats. Anesthesiology 76: 948-952, 1992.
36. Eikermann M, Vetrivelan R, Grosse-Sundrup M, Henry ME, Hoffmann U, Yokota S, Saper CB and Chamberlin NL: The ventrolateral preoptic nucleus is not required for isoflurane general anesthesia. Brain Res 1426: 30-37, 2011.

37. Zhang Y, Yu T, Yuan J and Yu BW: The ventrolateral preoptic nucleus is required for propofol-induced inhibition of locus coeruleus neuronal activity. Neurol Sci 36: 2177-2184, 2015.

38. Tung A, Szafran MJ, Bluhm B and Mendelson WB: Sleep deprivation potentiates the onset and duration of loss of righting reflex induced by propofol and isoflurane. Anesthesiology 97: 906-911, 2002.

39. Suntsova N, Szymusiak R, Alam MN, Guzman-Marin R and McGinty D: Sleep-waking discharge patterns of median preoptic nucleus neurons in rats. J Physiol 543: 665-677, 2002.

40. Szymusiak R and McGinty D: Sleep suppression following kainic acid-induced lesions of the basal forebrain. Exp Neurol 94: 598-614, 1986.

41. Gerashchenko D, Wisor JP, Burns D, Reh RK, Shiromani PJ, Sakurai T, de la Iglesia HO and Kilduff TS: Identification of a population of sleep-active cerebral cortex neurons. Proc Natl Acad Sci USA 105: 10227-10232, 2008. 